\title{
Healthy Aims: Developing New Medical Implants and Diagnostic Equipment
}

\author{
A European Union project consortium is developing intelligent medical \\ implants and diagnostic systems that integrate a range of underpinning \\ microprocessor, biological, wireless, and nano technologies.
}

Diana Hodgins

European Technology for Business

Arnaud Bertsch

Ecole Polytechnique Fédérale de Lausanne

Nils Post

Intelligent Medical Implants

Manfred Frischholz

Campus Micro Technologies

Bart Volckaerts

Cochlear Technology

Centre Europe

John Spensley

Finetech Medical

J.M. Wasikiewicz

University of London

Henry Higgins

Zarlink Semiconductor

Felix von Stetten

University of Freiburg (IMTEK)

Laurence Kenney

University of Salford

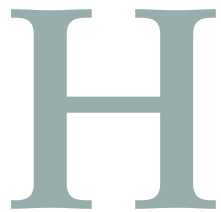

ealthy Aims is a $€ 23$ million, four-year project, funded under the EU's Information Society Technology Sixth Framework program to develop intelligent medical implants and diagnostic systems (www.healthyaims. org). The project has 25 partners from 10 countries, including commercial, clinical, and research groups. This consortium represents a combination of disciplines to design and fabricate new medical devices and components as well as to test them in laboratories and subsequent clinical trials.

The project focuses on medical implants for nerve stimulation and diagnostic equipment based on straingauge technology. Figure 1 shows several products in development:

- a retinal implant for restoration of sight,

- functional electrical stimulation (FES) systems for restoration of upper-limb movement as well as bladder and bowel control,

- a cochlear implant for restoration of hearing,

- a glaucoma sensor for diagnosing glaucoma,

- an intracranial pressure (ICP) sensor system for monitoring brain pressure,

- a sphincter sensor for monitoring bladder pressure, and

- an activity monitor and laboratory system for accurate interpretation of human motion.

These products have clear market applications, targeting citizens suffering from disabilities that affect their quality of life-for example, blindness, stroke disabilities, deafness, and incontinence. With an estimated 500 million citizens in the Western population suffering from at least one of the health problems targeted in this project, the potential market and impact on society are huge.

Healthy Aims has also developed core technologies in parallel with the products with a view to stimulating future medical-implant technology. These include implantable power sources, RF communications suitable for implanting in the human body, and biocompatible encapsulation materials. 
Figure 1. Schematic of the final Healthy Aims project products.

\section{Status of project products}

Now in its final year, the Healthy Aims projects have achieved some remarkable results in product development.

\section{Retinal implant}

The retinal implant system is suitable for patients suffering from retinitis pigmentosa, cone-rod dystrophy, and macular degeneration. All these diseases include progressive degeneration of the photoreceptors, which leads to complete blindness. However, the retinal ganglion cell layer often remains intact. The implant system uses this layer as the basis for conducting nerve signals to the brain's visual areas. ${ }^{1}$

The system consists of three main components:

- the retinal stimulator, which is implanted in the eye;

- the visual interface, worn as glasses; and

- the rechargeable pocket processor worn around the waist.

Figure 2 shows the retinal stimulator. It's a complex implant comprising 2D or 3D electrode arrays, which connect to the ganglion cells; interconnects from the electrodes to the implant electronics; implant electronics, including an ASIC for interpreting excitation pulses to individual electrodes; and IR power and data transmission.

The stimulator required a high-density array with individually addressable electrodes that can provide sufficient charge density to stimulate the ganglion cells. ${ }^{2}$ Figure 3 shows the platinum array designed by Intelligent Medical Implants (www.intmedimplants.de) and fabricated by ITE (www. ite.waw.pl). The array has 234 individually addressable electrode groups. Each group comprises a $3 \times 3$ array of truncated electrodes. Each electrode measures $60 \times 60 \mu \mathrm{m}$ to maximize the
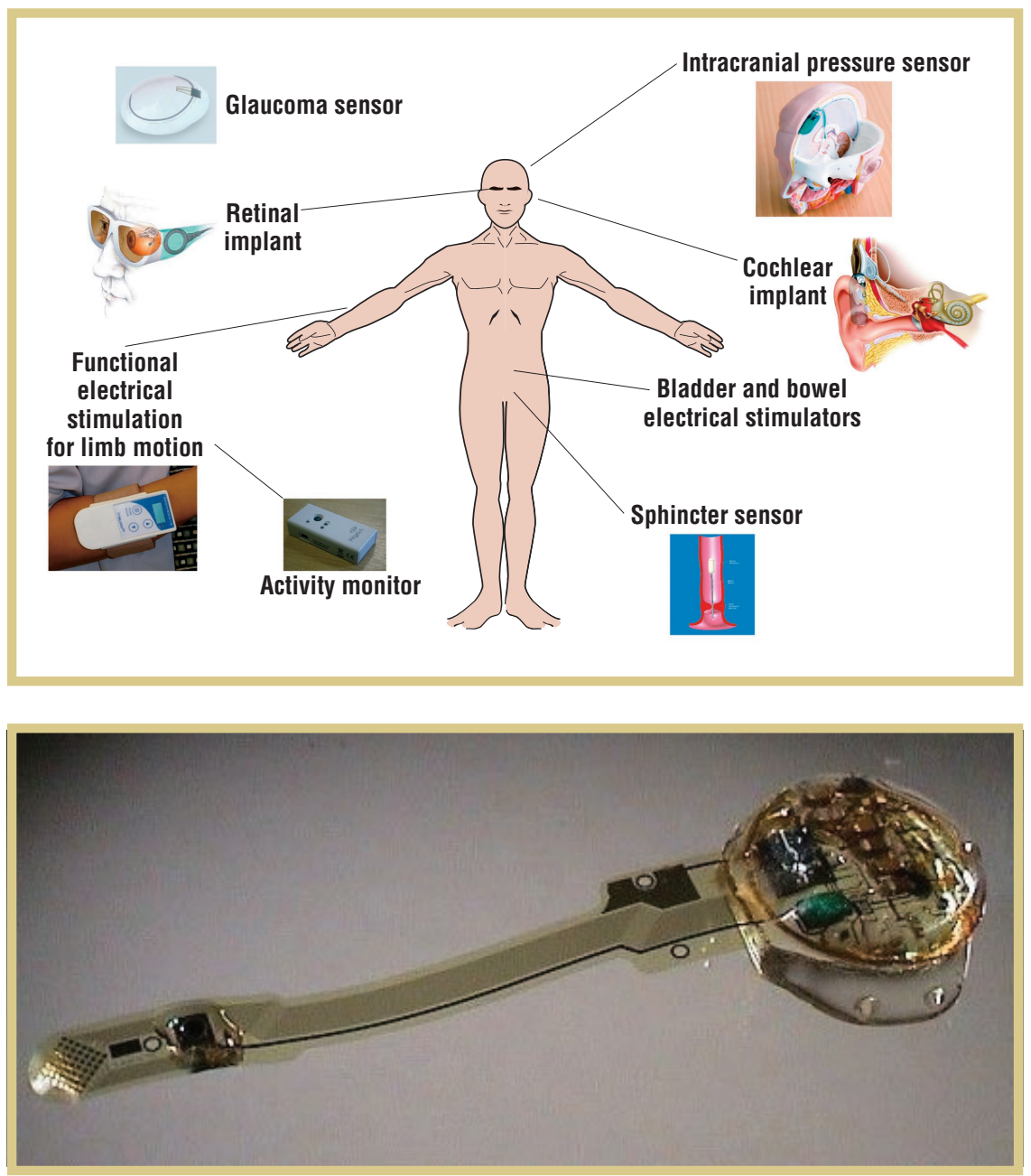

Figure 2. Retinal stimulator for the Healthy Aims retinal implant system. The electrode array is $4 \times 5.5 \mathrm{~mm}$, and the electronics is approximate $25 \mathrm{~mm}$ in diameter.

contact area on the ganglion cells. One block of $3 \times 3$ electrodes is $225 \times 225$ $\mu \mathrm{m}$, and the complete array, which is fixed to the retina, is approximately $3,900 \times 5,450 \mu \mathrm{m}$.

The retinal-stimulator electronics are located in the eye socket, and a biocompatible material encapsulates the entire implant.

The system's visual interface integrates a recording camera and image data transmission in a pair of glasses that connect to the pocket processor via cable. The pocket processor provides the power for the implant and on-thebody unit. It also contains a processor that determines the excitation pulses for the image's electrode array. The unit has programming capability from the PC via Ethernet.

The designed and fabricated system has successfully completed laboratory trials and is now undergoing human clinical trials at the Medical University of Graz.

\section{Upper limb FES implant}

This FES application has two development phases. In phase 1 , FineTech Medical implemented the STIMUGRIP system (www.finetech-medical.co.uk/producthand.htm). It includes a control system that's worn on the body, adjacent to the implant. The implant comprises two electrodes connected to passive electronics. The system helps patients with an 


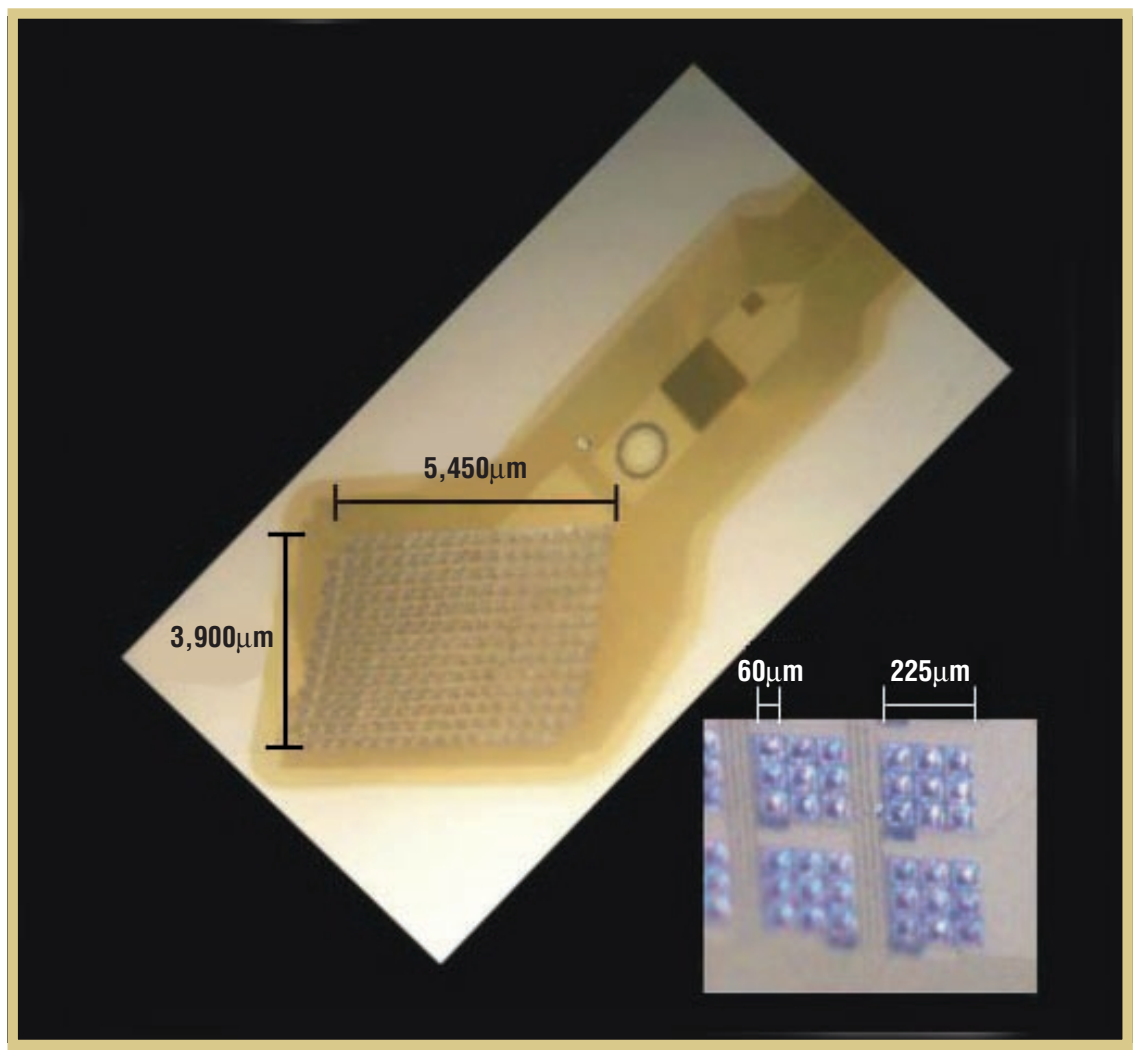

upper motor neuron lesion extend their wrist and open the hand.

Figure 4 shows the two parts: a passive, inductively powered, two-channel implanted stimulator and an external control unit. The external control unit incorporates batteries, an inductive coil that provides power to the implant, and control electronics. It also houses a three-axis accelerometer that detects arm movement. The accelerometer signals provide inputs to a state machine algorithm that determines the onset and termination of stimulation. FineTech Medical has developed software to automate and optimize the parameter setups for the state machine algorithm. ${ }^{3}$ The patient wears the controller unit on the affected limb, directly over the implant site. A strap secures it in place.

Phase 1 clinical trials are under way at the UK's Salisbury District Hospital, and initial results on the first two patients are promising.

In phase 2 , the project will implant the active components. This requires
Figure 3. A 3D retina electrode array with 234 individually addressable electrode groups.

gies, improve music appreciation and speech perception in noisy conditions. The consortium partners opted to build intracochlear electrodes that integrate with stimulation-site silicon chips and so bring the active stimulation circuitry to the inner cochlea. The interconnect technology is based on an intracochlear bus structure, which has the potential to increase the number of stimulation contacts significantly while eliminating the need for a separate, direct-wire connection from each stimulation site to the receiver-stimulator.

The fabrication process selected for these smart electrodes is based on wafer-level packaging techniques used in the semiconductor industry and developed by IMEC. ${ }^{4}$ The process integrates ASIC circuitry using waferto-wafer bonding, followed by wafer thinning and deep-reactive-ion etching to separate the individual ICs. The ICs are subsequently interconnected by way of platinum tracks and embedded in multiple barrier layers made from platinum, Parylene- $\mathrm{C}$, and silicone. These biocompatible materials prevent potentially harmful materials from migrating into the cochlea fluid. They also keep moisture from damaging the IC.

\section{Glaucoma sensor}

Changes in eye pressure is one symptom of the onset of glaucoma. The glaucoma sensor system noninvasively determines these changes for up to 24 hours. It comprises a contact lens with an embedded strain gauge that detects changes in curvature caused by changes in eye pressure. ${ }^{5}$

The strain gauge and subsequent electronic circuitry for producing an analog signal require power. The glaucoma sensor system uses a pair of spectacles and an associated body-worn unit to provide power over an inductively coupled circuit running at $27.3 \mathrm{MHz}$. The same inductive link also transmits the 
Figure 4. Upper-limb functional electrical stimulation system for restoring upper-limb movement: (a) implant electronics and electrodes and (b) external control unit.

analog signal. The body-worn power unit receives the signal and either stores the data for subsequent download and analysis or transmits it immediately via Bluetooth to a PC.

The consortium partners have now produced the first wireless contact lens version of the system, which is undergoing pilot clinical trials. It combines a 50- $\mu$ m-thick ASIC using the flip-chip attachment method, microfabricated strain gauges, and biocompatible coating materials. Considerable effort went into developing techniques for thinning ASICs and attaching them to flexible membranes. Figure 5a shows the contact lens, and figure $5 \mathrm{~b}$ shows the cross section of the ASIC assembly. Healthy Aims has applied and successfully demonstrated the thinning and flip-chip bonding techniques to not only the prototype glaucoma sensor but also the cochlear active implant and the ICP sensor system. The demonstration results show adequate compliance for these applications.

\section{Intracranial pressure sensor system}

The ICP sensor system is suitable for short- or long-term monitoring of pressure in the brain cavity. ${ }^{6}$ Short-term monitoring applies, for example, to people who have suffered head trauma in an accident and are in intensive care. Long-term monitoring applies to people suffering with hydrocephalus who are treated with a shunt for draining excessive fluid. In the latter application, surgeons would place the ICP system in the brain cavity at the same time as the shunt. If the shunt gets blocked, it can't remove the fluid and pressure therefore builds up in the brain. The sensor system detects this pressure increase.

Because the system is aimed at short-
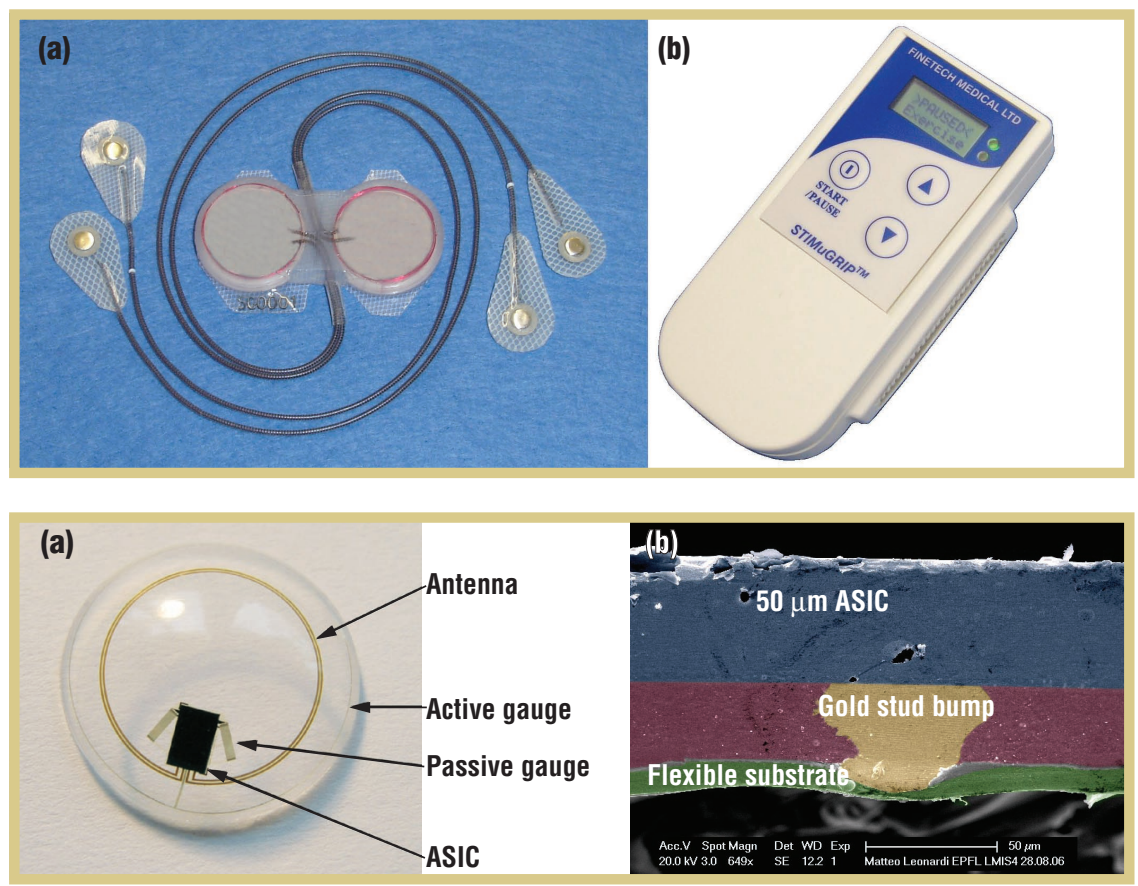

Figure 5. Glaucoma sensor (a) contact lens and (b) ASIC assembly.

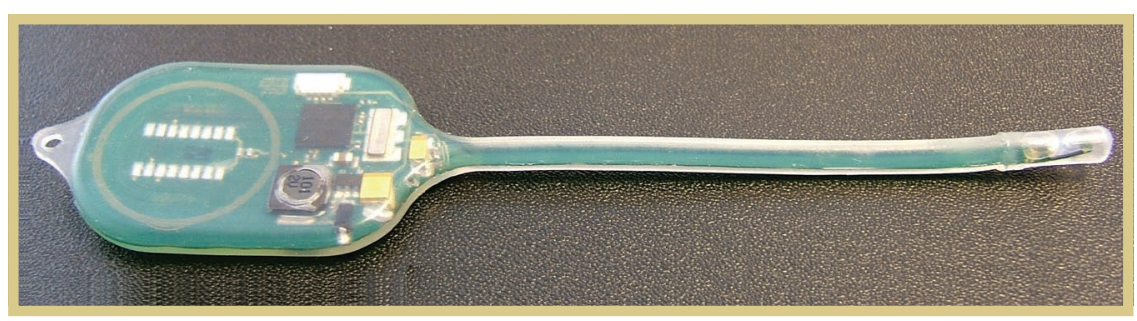

Figure 6. Fully implantable wireless sensor for the intracranial pressure monitoring system.

and long-term implant applications, the pressure sensor must be extremely stable and safe over time. The consortium partners have therefore completely encapsulated the implant with a biocompatible material and incorporated a stable, microfabricated, capacitivepressure sensor and associated electronics. A separate, external RF reader powers the unit and receives the pressure readings from the implant.

Figure 6 shows the implant. The very small pressure-sensor head (diameter $3 \mathrm{~mm}$ ) is located in the brain cavity and connected to the RF communication electronics, both mounted on the same flexible substrate. The associated electronics and circuitry for inductive- coupling power and data transmission are mounted on an enlarged part of the substrate, which is located outside the skull where space isn't a limiting factor. The system receives its power from the handheld external reader and transmits data at $13.56 \mathrm{MHz}$ (similar to RFID technology). Readings can be taken as often as required. This might be daily for long-term users, whereas it could be several readings per second for people in intensive care. The system can store data in the reader (an integrated data logger), or it can transmit data directly via $\mathrm{ZigBee}$ to a PC for further analysis.

Campus Micro Technologies (www. campus-micro-technologies.de) has produced ICP prototypes, which have 
TABLE 1

Implantable lithium-ion battery characteristics.

\section{Characteristic \\ Characteristic}

\begin{tabular}{l|c} 
Operating temperature & $37^{\circ} \mathrm{C}$ \\
\hline Dimensions & $<5 \times 10 \times 22 \mathrm{~mm}$ \\
\hline Weight & $2.45 \mathrm{~g}$ \\
\hline Capacity & $50 \mathrm{mAh}$ \\
\hline Lifetime at $37^{\circ} \mathrm{C}$ & $>10 \mathrm{yrs}$ \\
\hline Number of charges/discharges* & $>4,000$ \\
\hline Autonomy & $16-20 \mathrm{hrs}$ \\
\hline Minimum charge time & $2 \mathrm{hrs}$ \\
\hline Voltage window & $3.0-4.0 \mathrm{~V}$ \\
\hline Maximum continuous discharge current & $10 \mathrm{~mA}$ \\
\hline Maximum pulse $(1000 \mu \mathrm{s})$ current & $50 \mathrm{~mA}$ \\
\hline $100 \%$ depth of discharge, with more than $50 \%$ of initial capacity remaining.
\end{tabular}

shown excellent stability over a 30-day period in lab trials. Thirty days is the critical performance requirement for short-term applications. Animal trials are now planned for the final year at the UK North Bristol National Health System's Neurosurgery Department (www.nbt.nhs.uk/services/neurosciences/neurosurgery.htm).

\section{Sphincter sensor}

The sphincter sensor is used in a urodynamics procedure to diagnose the cause for urinary incontinence. The sphincter sensor is inserted into the bladder and monitors dynamic bladder pressure over time. Currently, the urodynamics procedure involves forcefilling the bladder with water and measuring pressure changes as the patient empties it. Future iterations of the product will enable an ambulatory test, which isn't used regularly now because the cost is prohibitive. In the ambulatory test, the sensor remains in the body for 24 hours, measuring pressure as the patient fills and voids the bladder.

For both applications, the consortium partners have developed a low-cost microfabricated strain gauge mounted on a catheter. The strain gauge is fabricated on an $8-\mu \mathrm{m}$ film that is mounted in the catheter tip and deforms under pressure. Wires run from the sensor along the catheter where they interface into a data-logging unit.

Laboratory trials on the sensor assembly have shown that it achieves the required resolution of $1 \mathrm{~cm}$ of water with a maximum pressure of $1.5 \mathrm{~m}$. It can also log data over the required 24-hour period. Laboratory trials in a simulated bladder environment with the full catheter assembly and logging system will take place within six months.

\section{Activity monitor}

A growing body of research has linked physical inactivity to many common diseases such as cardiovascular disease hypertension, diabetes mellitus, and obesity. These studies typically rely on self-reported activity levels, but evidence suggests that this isn't a reliable way to accurately quantify physical activity patterns. In many cases, using an automated activity-monitoring system provides direct measurement of the type, intensity, and duration of activity over an extended period. Healthy Aims wanted to develop a measurement unit, together with a range of software for activity classification and stride parameter estimation, that would improve on the performance of currently available systems. Current systems restricted specificity and, in some cases, required users to wear sensors on multiple limb segments.
European Technology for Business is a specialist microsystem design house that focuses on medical applications. ETB developed the activity monitor hardware in this project. It incorporates a three-axis accelerometer. It can run for up to 24 hours, storing data directly onto a memory card. At the activity's end, the monitor downloads the data to the PC for analysis using algorithms developed within the project.

ETB has also developed algorithms for monitoring stride characteristics, including stride frequency and stride length, from a single waist-worn activity monitor. The University of Salford's Healthy Aims group is developing algorithms for classifying activity type on the basis of a nearest-neighbor technique. Initial results suggest that the adopted classification approach outperforms other similar approaches (for a review of accelerometry-based activity classification approaches, see Merryn Mathie and colleagues ${ }^{7}$ ).

The University of Salford group has conducted trials on 10 healthy and 10 obese subjects. The trial protocol included a period on the treadmill followed by a range of outdoor activities including hopping, stair climbing, walking, jogging, and running. Participants wore monitors on their waist, thighs, and shanks to enable a systematic appraisal of how the number and location of monitors affected algorithm performance.

\section{Key technologies}

Developments in three core technologies supported the Healthy Aims products.

\section{Implantable battery}

The requirements for implant power sources are extremely challenging. Saft and the CEA designed a $50 \mathrm{mAh}$ prismatic rechargeable lithium-ion cell that meets these requirements in a range of 
Figure 7. Schematic diagrams of a surface-mountable glucose biofuel cell: (a) cross section and (b) magnified view of the electrode structure.

nonvital medical-device applications. In addition to high energy density and a proven record of long cycle and calendar life, the battery meets the key requirement of not significantly raising the body tissue temperature while charging. Table 1 lists some of its detail characteristics.

Healthy Aims selected it for the cochlear implant and FES systems. Work is now under way to integrate the battery and charging circuit into the implant enclosure for these applications.

\section{Biofuel cell}

Some implant applications-for example, a pacemaker-have low enough power requirements to use for a biofuel cell. Healthy Aims has developed a direct biofuel cell that derives electricity directly from body fluids. Specifically, it uses the spatially separated electrochemical reaction of glucose and oxygen.

The cell has a selective cathode consisting of activated carbon that's arranged in front of the anode. ${ }^{8}$ The carbon effectively depletes oxygen inside the fuel cell, and glucose oxidation takes place under predominantly anoxic conditions. This concept enables the fuel cell to be mounted directly on the surface of medical implants, facilitating system integration and implantation.

Figure 7 shows the cell's construction. The electrode dimensions are $1.5 \mathrm{~cm} \times 1.5 \mathrm{~cm}$. To increase electrical conductivity, both electrodes include a platinum-mesh electron collector. Platinum wires establish electrical connections to the electron collector with conductive carbon cement. The electrodes are electrically insulated by a glucosepermeable membrane. An epoxy resin casing gives structural stability to the membrane electrode assembly.

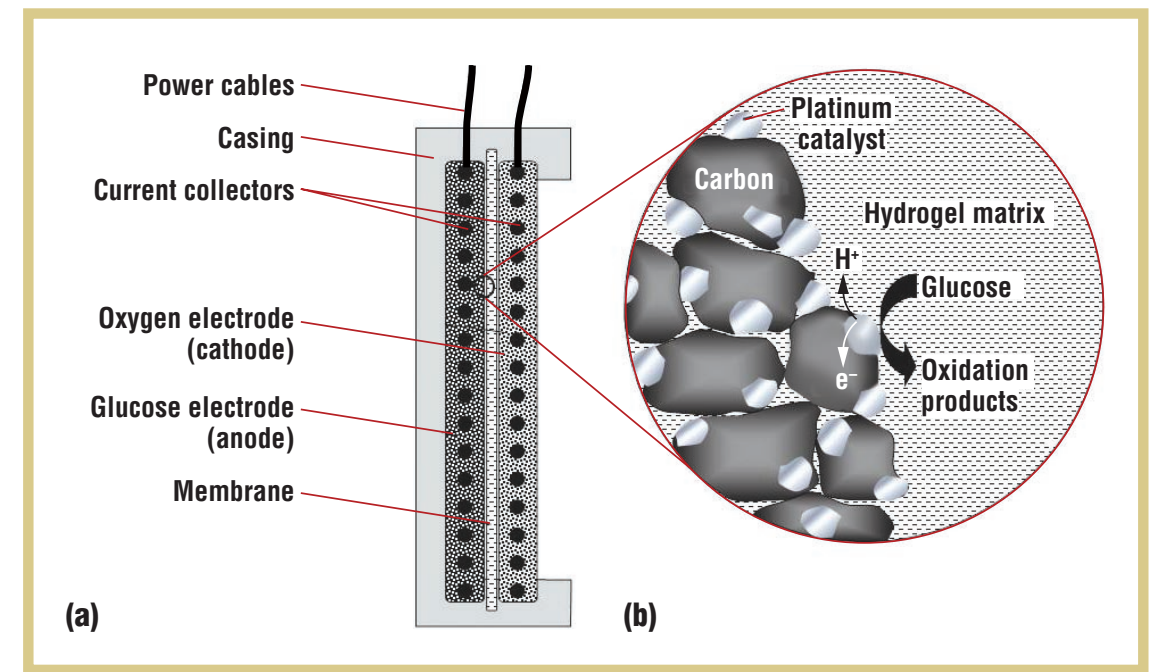

The consortium partners, University of Freiburg (IмTEK) and University of Rovira i Virgili (DinAMIC), characterized the fuel cell's electrochemistry in tests carried out at $37^{\circ} \mathrm{C}$ in $600 \mathrm{ml}$ of aerated phosphate-buffered saline solution ( $\mathrm{pH}$ 7.4) containing 0.1 percent glucose by weight (the appoximate concentration of human interstitial fluid). A stable power output of $1.5 \mu \mathrm{W} / \mathrm{cm}^{2}$ was observed after 200 days, with a corresponding cell voltage of $112 \mathrm{mV}$.

The results demonstrate that a $3 \mathrm{~cm} \times$ $3 \mathrm{~cm}$ biofuel cell, applied to the surface of an implant, would be sufficient to power an $8 \mu \mathrm{W}$ pacemaker circuit. The concept thus shows promise for developing autonomous, sustainable power supply systems for long-term medical implants.

\section{Wireless communication}

Because implanted devices must be completely self-contained, the data transfer method from inside the body must be wireless. Application requirements and medical safety standards define the data-transfer carrier frequency.

$\mathrm{RF}$ technology supports suitable wireless links between implants and bodyworn units when power is available within the implant. For Healthy Aims' FES and cochlear-implant projects, Zarlink has integrated a wake-up function into a medical-implant RF transceiver (http://assets.zarlink.com/PP/ ZL70101_Product_Preview_0907.pdf).
The wake-up function leaves the RF circuit turned off for most of the time.

The frequency assigned for implanteddevice RF communication is around $403 \mathrm{MHz}$ and referred to as the MICS (Medical Implant Communication System) band.

The 2.4 to $2.5 \mathrm{GHz}$ ISM (Industrial Scientific and Medical) band is suitable for transmitting to an implant but not from it. Transmitting the wake-up signal to an implant on the ISM band requires less current in the receiver design than transmitting on the MICS band would. The average current consumption decreases further if the receiver is only switched on for short periods of time at predetermined intervals. If the strobe frequency is once per second with an on time of $240 \mathrm{~ns}$, the wake-up receiver's average current is typically $200 \mathrm{nA}$.

Once the implant receives a valid wake-up signal, the remainder of its implant RF circuit can be powered up within $20 \mathrm{~ms}$. The transceiver remains powered for the duration of the interrogation and then shuts off until it receives another wake-up signal. In this way, the communication circuit uses significant current only during data transfers.

Transferring the data at a high speed further minimizes the wake-up time for the implant transceiver. Typically, a raw data rate of $200 \mathrm{Kbps}$ to $800 \mathrm{Kbps}$ is possible. 


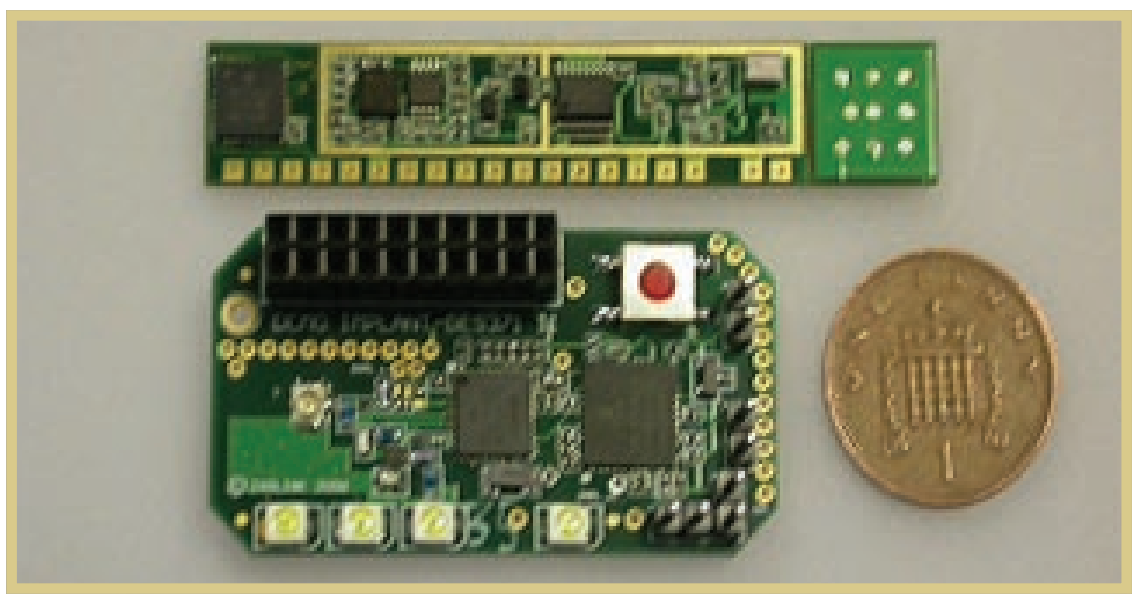

Zarlink has integrated the MICS chip into two FES implants, one for upper arm movement and the other for bladder control. University College London and Finetech Medical are now working to integrate the chip and antenna into the implant enclosure. Figure 8 shows a demonstrator, now available from Zarlink, that includes a processor to prove the communication link. For more details on this project, see Body Sensor Networks, chapter 4.

\section{Biocompatible implant coatings}

All implants will be completely encapsulated with a biocompatible material to prevent leaching from implant components to the body and to stop water ingress to the components. These biocompatible coatings must remain stable for the implant's lifetime, which in many cases can be the same as a person's lifetime. Healthy Aims researchers at Queen Mary, University of London have been working with the implant product partners to develop biocompatible coatings that are thin, flexible, and rugged enough for handling during surgery. ${ }^{9}$ Preliminary results using lipidmodified silicone and diamond-like carbon (DLC) indicate that these materials, both together and individually, could meet the mechanical requirements and improve the water resistance compared to silicone alone. ${ }^{10}$

Applying DLC as a very thin coating (100 nm or less) directly on a sensor's surface doesn't significantly affect per- formance. It can also be applied as a sandwich layer with conventional materials such as Parylene-C or silicone.

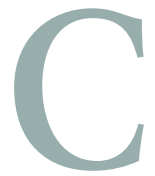

ollectively, the Healthy Aims project has stimulated the development of medical implant and diagnostic equipment. Human clinical trials are now successfully underway for the two-channel, upper-arm FES system as well as the glaucoma sensor and the activity monitor. Acute animal trials on the FES system for bowel control have concluded successfully, and plans for chronic trials are underway. Laboratory trials for the cochlear implant's active electrodes have begun, and Cochlear Technology Centre has successfully concluded initial trials on the prototype implant battery.

The core technologies developed to support these and other Healthy Aims projects will further advance this growing field of medical technology. I P

\section{ACKNOWLEDGMENTS}

The Healthy Aims partnership thanks the European Commission for IST FP6 funding, without which these achievements wouldn't have been possible.

\section{REFERENCES}

1. R. Hornig et al., "A Method and Technical Equipment for an Acute Human Trial to Evaluate Retinal Implant Technology," J. Neural Eng., vol. 2, no. 1, 2005, pp. S129-S134.
Figure 8. Elements of the Medical Implant Communication Service demonstrator.

2. T.A. Fofonoff et al., "Microelectrode Array Fabrication by Electrical Discharge Machining and Chemical Etching," IEEE Trans. Biomedical Eng., vol. 51, no. 6, 2004, pp. 890-895.

3. P. Tresadern et al., "A Clinical Set-up Tool (CST) for Rapid Stimulator Programming," Proc. 29th IEEE Ann. Int'l Conf. Eng. Medicine and Biology Society (EMBS 07), IEEE Press, 2007, pp. 35773580 .

4. M. Vanden Bulcke et al., "Active Electrode Arrays by Chip Embedding in a Flexible Silicone Carrier," Proc. 28th IEEE Ann. Int'l Conf. Eng. Medicine and Biology Society (EMBS 06), IEEE Press, 2006, pp. 2811-2815.

5. M. Leonardi et al., "A Soft Contact Lens with a MEMS Strain Gage Embedded for Intraoccular Pressure Monitoring," Proc. 12th Int'l Conf. Transducers, Solid-State Sensors, Actuators, and Microsystems (Transducers 03), IEEE Press, 2003, pp. 1043-1046.

6. T. Eggers and M. Wenzel, "Implantable Telemetric Pressure Measurement," Medical Device Technology, vol. 15, no. 4, 2004.

7 M.J. Mathie et al., "Accelerometry: Providing an Integrated, Practical Method for Long-Term, Ambulatory Monitoring of Human Movement," Physiological Measurement, vol. 25, no. 2, 2004, pp. R1-20.

8. F. von Stetten et al., "A One-Compartment, Direct Glucose Fuel Cell for Powering Long-Term Medical Implants," Proc. 19th IEEE Int'l Conf. Micro Electro Mechanical Systems (MEMS 06), IEEE Press, 2006, pp. 934-937.

9. S.M. Reddy and P.M. Vadgama, "Ion Exchanger Modified PVC MembranesSelectivity Studies and Response Amplification of Oxalate and Lactate Enzyme Electrodes," Biosensors and Bioelectronics, vol. 12, nos. 9-10, 1997, pp. 10031012.

10. J. Franks, T.L. Ng, and A.C. Wright, "Preparation and Characteristics of Diamond-Like Carbon Films," Vacuum, vol. 38, nos. 8-10, 1988, pp. 749-751.

11. H. Higgins, "Wireless Communication," Body Sensor Networks, G.Z. Yang, ed., Springer, 2006, pp. 117-144. 


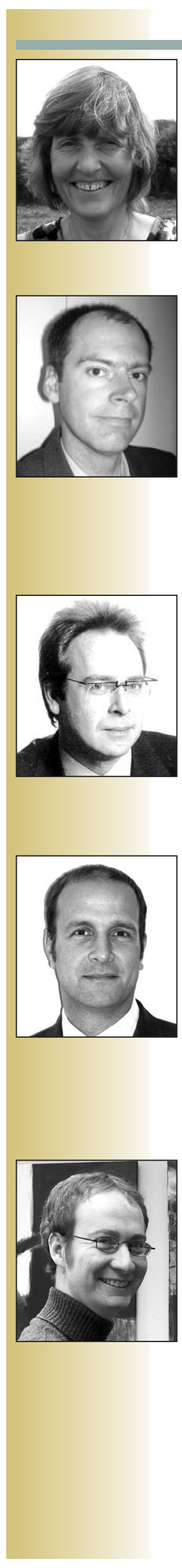

Diana Hodgins is managing director of European Technology for Business and project coordinator for the Healthy Aims project. Her research interests include microsystem technology and design. She received her $\mathrm{PhD}$ in solid-state physics from the University of Hertfordshire. Contact her at ET B, Codicote Innovation Centre, St Albans Rd., Codicote, Herts, SG4 8WG, UK; dmh@etb.co.uk, www. etb.co.uk.

Arnaud Bertsch is a senior scientist at the Ecole Polytechnique Fédérale de Lausanne. His research interests include polymer and nanocomposites for microfabrication, MEMS for medicine, bioMEMS, and applied nanofluidics. In the Healthy Aims project, he was involved in developing the glaucoma sensor. He received his $\mathrm{PhD}$ in process engineering from the Institut National Polytechnique de Lorraine. Contact him at the Ecole Polytechnique Fédérale de Lausanne, STI-LMIS4, BM 3.124, Station 17, 1015 Lausanne, Switzerland; arnaud.bertsch@ epfl.ch.

Nils Post is a study coordinator at Intelligent Medical Implants. His research interests center on medical device development. He supervises the IMI's Retinal Implant System work package for the Healthy Aims project in general and especially in concerns of the clinical validation. He received his $\mathrm{PhD}$ in biology from Friedrich-Wilhelms University. Contact him at Clinical Validation, IMI, Niebuhrstraße 1a, D - 53113 Bonn, Germany; post@intmedimplants.de; www.intmedimplants.de.

Manfred Frischholz is managing director of Campus Micro Technologies. His research interests include sensor development and systems design for industrial and medical applications-in particular, the integration of MEMS and wireless technology for application in medical implants. He received his $\mathrm{PhD}$ in physics from the University of Erlangen-Nürnberg. Contact him at Campus Micro Technologies, Universitätsallee 29, D-28359 Bremen, Germany; manfred.frischholz@campusmicro-technologies.de; www.campus-microtechnologies.de.

Bart Volckaerts is a technology research coordinator at the Cochlear Technology Centre, where he coordinates research projects relating to micro- and nano technologies, implantable electronics, and biocompatible materials. His research interests include micro-optical and mechanical component fabrication for optical-fiber application integration. He received his $\mathrm{PhD}$ in micro-optics and photonics from the Vrije Universiteit Brussel. He is the spokesperson for CTC in the Healthy Aims project. Contact him at CTC Europe, Mechelen Campus, Schaliënhoevedreef 20 I, B-2800 Mechelen, Belgium; bvolckaerts@cochlear.be; www.cochlear.com.

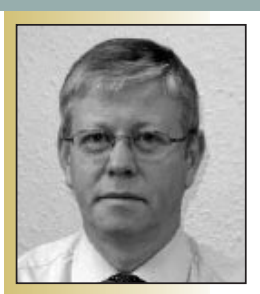

John Spensley is managing director of FineTech Medical, which manufactures medical devices under the Active Medical Device Directive 90/385/EEC, including long-term implantable neuromuscular stimulators. His research interests include manufacturing engineering and operations management. He received his MSc in technology management from Luton University. Contact him at Finetech Medical, 13 Tewin Ct., Welwyn Garden City, Hertfordshire AL7 1AU, UK; john.spensley@ finetech-medical.co.uk.

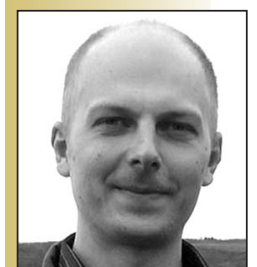

J.M. Wasikiewicz is a postdoctoral research assistant at Queen Mary University of London. His research interests include development of polymeric biomaterials as well as radiation modification of polysaccharides. He has a $\mathrm{PhD}$ in production engineering from Gunma University, Japan. Contact him at the Interdisciplinary Research Centre in Biomedical Materials, Queen Mary University of London, Mile End Rd., London, E1 4NS, UK; j.m.wasikiewicz@qmul.ac.uk; www.materials.qmul. ac.uk/irc.

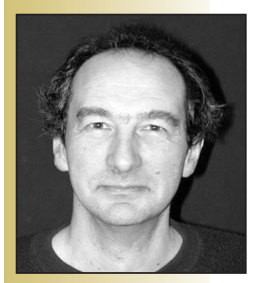

Henry Higgins is an electronic/RF design engineer with Zarlink Semiconductor, where he works on the design of RF systems to communicate from an implant within the body. His research interests include circuit design, simulation, layout, and testing in various environments as well as small-module design for implants. He has an MSc in microwave oscillator design from the University of Bath. Contact him at Advanced Packaging Business, Zarlink Semiconductor, Phase 2, Castlegate Business Pk., Caldicot, Monmouthshire, NP26 5YW, UK; henry. higgins@zarlink.com.

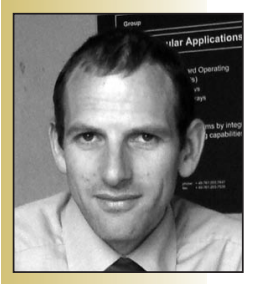

Felix von Stetten is Research Group Leader at the Laboratory for MEMS Applications, Department of Microsystems Technology (IMTEK), University of Freiburg. His research is dedicated to implantable biofuel cells and diagnostic lab-on-a-chip systems. He received his PhD in microbiology from the Technical University Munich. Contact him at the Laboratory for MEMS Applications, Dept. of Microsystems Eng. (IMTEK), Univ. of Freiburg, Georges-KoehlerAllee 106, D-79110 Freiburg, Germany; vstetten@ imtek.de; www.imtek.de/anwendungen.

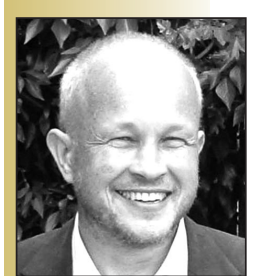

Laurence Kenney is a senior research fellow with the Centre for Rehabilitation and Human Performance Research at the University of Salford. His research focuses on the development and evaluation of rehabilitation technologies, particularly functional electrical-stimulation systems. He received his $\mathrm{PhD}$ in engineering design from the University of Salford and is a member of the Institute of Physics and Engineering in Medicine. Contact him at the School of Health Care Professions, Brian Blatchford Bldg., Univ. of Salford, Salford, M6 6PU, UK; I.p.j.kenney@salford.ac.uk; www.ihscr. salford.ac.uk/CRHPR. 